\title{
Global exponential stability of a class of impulsive recurrent neural networks with proportional delays via fixed point theory ${ }^{\star}$
}

\author{
Liqun Zhou*, Yanyan Zhang \\ School of Mathematics Science, Tianjin Normal University, Tianjin, 300387, \\ China
}

\begin{abstract}
In this paper, we study the stability of a class of impulsive recurrent neural networks with proportional delays. Here the proportional delay is a kind of unbounded variable delay, which is different from others delays. By using the fixed point theory and some analysis techniques, the stability of the system is investigated, two novel sufficient conditions are obtained to ensure the existence and uniqueness of a solution and the global exponential stability of the considered system. The results obtained in this paper extend and improve the results in previous literature. An example and its simulations are given to illustrate the effectiveness of the proposed results.
\end{abstract}

Key words: Recurrent neural networks (RNNs); Proportional delay; Fixed point theory; Global exponential stability; Impulse

\section{Introduction}

Recurrent neural networks (RNNs), for instance, cellular neural networks, Hopfield neural networks and Cohen-Grossberg neural networks, have received considerable attention for their potential applications in various fields, such as associative memory, image processing, pattern recognition and computer vision, etc. And these applications mainly depend on the stability of these neural networks, which is also a crucial property in the design of the neural

* This work is supported by the National Science Foundation of China (No.61374009).

* Corresponding author. Email address: zhouliqun20000@163.com. 
networks. The time delay is inevitable due to the finite switching speed of information processing and the inherent communication time of neurons, and its existence is frequently a source of oscillation and instability of the system. Thus, various stabilities of DRNNs have been widely studied and a great deal of results have been obtained (see, [1-6]). In fact, besides delay effects, stochastic, impulsive and diffusion effects are also likely to exist in the neural networks [7-11]. Thus, the dynamics behavior research of complex DRNNs, such as impulsive DRNNs, are discussed, and a large number of research results are obtained [16-20,23,24,37,38,40].

In general, the time delays considered for RNNs can be classified as constant delays $[5,17,23,24]$, time-varying delays $[2-4,12-16,18,19,21,37]$, and distributed delays $[1,6,20,22]$. As is known to all, the proportional delay is one of delay types and objective existence, for example the proportional delay is usually required in Web quality of service (QoS) routing algorithm. The proportional delay function is a kind of time-varying and unbounded delay which is different from the above mentioned delays. According to the topological structure of neural networks, the proportional delay is introduced in the neural networks, which is feasible. At present, the results of stability of RNNs with proportional delays have been reported in [25-31]. Dissipativity of a class of cellular neural networks $(\mathrm{CNNs})$ with proportional delays was investigated by using the inner product properties in [25]. In [26-28], Zhou had discussed the global exponential stability of CNNs with multi-proportional delays by employing matrix theory and constructing Lyapunov functional, respectively. Delay-dependent exponential synchronization of recurrent neural networks with multiple proportional delays was studied in [29] by constructing appropriate Lyapunov functional. In [30], stability criteria for high-order networks with proportional delay was studied based on matrix measure, Lyapunov functional and Halanay inequality. In [31], new explicit conditions ensuring that the state trajectories of the system do not exceed a certain threshold over a pre-specified finite time interval were obtained by matrix inequalities. The advantage of RNNs with proportional delays is that the network's running time can be controlled according to the network allowed delays. Thus, it is not only theoretically interesting but also practical to establish sufficient conditions for stability of neural networks with proportional delays.

So far, most stability results for DRNNs mainly depend on constructing appropriate Lyapunov functionals. It is well known that the fixed Lyapunov functional construction method has not been found. It is inspiring that in recent years, the stabilities of some systems are investigated by applying fixed point theory and some more applicable results are obtained [32-36,39]. Fixed point theory is employed to deal with the stabilities of stochastic (delayed) differential equations and other types of equations in [32-36,39]. In addition, fixed point theory also provides a new research method to study the stability of complex neural networks $[37,38]$. In [37], fixed point theory was used to in- 
vestigate the global exponential stability of impulsive cellular neural networks with time-varying delays. By using fixed point theory and some analysis techniques, exponential stability in mean square of a class of stochastic delayed neutral cellular neural network was studied in [38].

To the best of our knowledge, the stability of recurrent neural networks with impulse and proportional delays has not been reported by using fixed point theory. Motivated by the above discussion, in this paper, the global exponential stability of impulsive recurrent neural networks with proportional delays is discussed by applying fixed point theory and some analysis techniques. Without constructing the new Lyapunov functional, linear matrix inequality, or other troublesome methods in [25-31], we can directly study the neural networks with proportional delays, which is this method's advantage, and we improve the research methods in [37]. Moreover, some new and concise algebraic criteria are provided and easy to be verified.

The rest of the paper is organized as follows. In Section 2, models and preliminaries are presented. A class of recurrent neural networks with impulse and proportional delays is introduced. Global exponential stability of the equilibrium point of the system are investigated by using the fixed point theory and some analysis techniques in Section 3. In Section 4, a numerical example is given to illustrate the effectiveness of the proposed method. Conclusions are presented in Section 5.

\section{Model description and Preliminaries}

Throughout this paper, the following notation will be used. $\mathbb{R}^{n}$ denotes the $n$-dimensional Euclidean space; $\|\cdot\|$ represents the Euclidean norm; $\mathcal{N}=$ $\{1,2, \cdots, n\} ; C[X, Y]$ corresponds to the space of continuous mappings from the topological space $X$ to the topological space $Y$.

Consider the following impulsive recurrent neural networks with proportional delays

$$
\begin{aligned}
& \dot{x}_{i}(t)=-d_{i} x_{i}(t)+\sum_{j=1}^{n} a_{i j} f_{j}\left(x_{j}(t)\right)+\sum_{j=1}^{n} b_{i j} g_{j}\left(x_{j}\left(q_{j} t\right)\right), t \geq 1, t \neq t_{k}, \\
& \triangle x_{i}\left(t_{k}\right)=x_{i}\left(t_{k}+\right)-x_{i}\left(t_{k}\right)=P_{i k}\left(x_{i}\left(t_{k}\right)\right), k=1,2, \cdots \\
& x_{i}(s)=x_{i 0}, s \in[q, 1], q=\min _{0 \leq j \leq n}\left\{q_{j}\right\}
\end{aligned}
$$

for $i \in \mathcal{N}$. Where $n$ means the dimension of system. $x_{i}(t)$ denotes the state of the $i$ th neuron at time $t$. The constant $d_{i}>0$ describes the rate with which the $i$ th neuron will reset its potential to the resting state in isolation when 
disconnected from the networks and external inputs. $f_{j}(\cdot), g_{j}(\cdot) \in C[\mathbb{R}, \mathbb{R}]$, $f_{j}\left(x_{j}(t)\right)$ and $g_{j}\left(x_{j}\left(q_{j} t\right)\right)$ represent the output functions of the $j$ th neuron at time $t$ and $q_{j} t$, respectively. The constants $a_{i j}$ and $b_{i j}$ denote the connection weight of the $j$ th neuron to the $i$ th neuron at time $t$ and $q_{j} t$, respectively. $q_{j}$ is a proportional delay factor and satisfies $0<q_{j} \leq 1$, and $q_{j} t=t-\left(1-q_{j}\right) t$, in which $\left(1-q_{j}\right) t=\tau(t)$ is a time-varying continuous function that satisfies $\left(1-q_{j}\right) t \rightarrow+\infty$, as $t \rightarrow+\infty$. The fixed impulsive moments $t_{k}(k=1,2, \cdots)$ satisfy $1=t_{0}<t_{1}<t_{2}<\cdots$, and $\lim _{k \rightarrow \infty} t_{k}=\infty . x_{i}\left(t_{k}+\right)$ and $x_{i}\left(t_{k}-\right)$ stand for the right-hand and left-hand limit of $x_{i}(t)$ at time $t_{k}$, respectively. $P_{i k}\left(x_{i}\left(t_{k}\right)\right)$ shows the abrupt changed of $x_{i}(t)$ at the impulsive moment $t_{k}$ and $P_{i k} \in C[\mathbb{R}, \mathbb{R}]$. Furthermore, $x_{i}(s)=x_{i 0}, s \in[q, 1]$, denotes the initial conditions, where $x_{i 0}, i=1,2, \cdots, n$ are constants and $x_{0}=\left(x_{10}, x_{20}, \cdots, x_{n 0}\right)^{T}$. Throughout this paper, we always assume that $f_{i}(0)=g_{i}(0)=P_{i k}(0)=0$ for $i \in \mathcal{N}$ and $k=1,2, \cdots$.

Denote by $x(t)=x\left(t ; s, x_{0}\right)=\left(x_{1}\left(t ; s, x_{10}\right), x_{2}\left(t ; s, x_{20}\right), \cdots, x_{n}\left(t ; s, x_{n 0}\right)\right)^{T} \in$ $\mathbb{R}^{n}$ the solution to systems (2.1)-(2.3). The solution $x(t)=x\left(t ; s, x_{0}\right) \in \mathbb{R}^{n}$ is, for the time variable $t$, a piecewise continuous vector-valued function with the first kind discontinuity at the points $t_{k}(k=1,2, \cdots)$, where it is leftcontinuous, i.e., the following relations are valid:

$$
x_{i}\left(t_{k}-\right)=x_{i}\left(t_{k}\right), x_{i}\left(t_{k}+\right)=x_{i}\left(t_{k}\right)+P_{i k}\left(x_{i}\left(t_{k}\right)\right), i \in \mathcal{N}, k=1,2, \cdots .
$$

The nonlinear output functions $f_{i}\left(x_{i}(\cdot)\right), g_{i}\left(x_{i}(\cdot)\right)$ and the impulsive moment $P_{i k}(\cdot)$ are assumed to satisfy the following conditions:

There exist nonnegative constants $l_{j}, k_{j}, p_{j k}, j \in \mathcal{N}, k=1,2, \cdots$, such that for any $u, v \in \mathbb{R}$,

$$
\begin{aligned}
& \left|f_{j}(u)-f_{j}(v)\right| \leq l_{j}|u-v|, \\
& \left|g_{j}(u)-g_{j}(v)\right| \leq k_{j}|u-v|, \\
& \left|P_{j k}(u)-P_{j k}(v)\right| \leq p_{j k}|u-v| .
\end{aligned}
$$

Definition 2.1 Systems (2.1)-(2.3) are said to be globally exponentially stable, if for any initial value vector $x_{0} \in C\left([q, 1] ; \mathbb{R}^{n}\right)$, there exists two positive constants $\lambda$ and $M$ such that

$$
\left\|x\left(t, s, x_{0}\right)\right\| \leq M e^{-\lambda t}, t \geq 1 .
$$

Theorem 2.2 [41] Let $\gamma$ be a contraction operator on a complete metric space $\Theta$, then there exists a unique point $\xi \in \Theta$ for which $\gamma(\xi)=\xi$.

Let $\mathcal{H}=\mathcal{H}_{1} \times \mathcal{H}_{2} \times \cdots \times \mathcal{H}_{n}$, and let $\mathcal{H}_{i}(i \in \mathcal{N})$ be the space consisting of functions $\phi_{i}(t):[q, \infty) \rightarrow \mathbb{R}$, where $\phi_{i}(t)$ satisfies:

(1) $\phi_{i}(t)$ is continuous on $t \neq t_{k}(k=1,2, \cdots)$; 
(2) $\lim _{t \rightarrow t_{k}^{-}} \phi_{i}(t)$ and $\lim _{t \rightarrow t_{k}^{+}} \phi_{i}(t)$ exist; furthermore, $\lim _{t \rightarrow t_{k}^{-}} \phi_{i}(t)=\phi_{i}\left(t_{k}\right)$ for $k=$ $1,2, \cdots$

(3) $\phi_{i}(s)=\varphi_{i}(s)$ on $s \in[q, 1]$;

(4) $\mathrm{e}^{2 \alpha t} \phi_{i}(t) \rightarrow 0$ as $t \rightarrow \infty$, where $\alpha$ is a positive constant and satisfies $\alpha<$ $\frac{1}{2} \min _{i \in \mathcal{N}}\left\{d_{i}\right\}$, here $t_{k}(k=1,2, \cdots)$ and $\varphi_{i}(s)=x_{i 0}, i=1,2, \cdots, n, s \in[q, 1]$ are defined as shown in (2.2)-(2.3). Also, $\mathcal{H}$ is a complete metric space when it is equipped with a metric defined by

$$
d(h(t), \bar{h}(t))=\sum_{i=1}^{n} \sup _{q \leq t<\infty}\left|h_{i}(t)-\bar{h}_{i}(t)\right|,
$$

where $h(t)=\left(h_{1}(t), h_{2}(t), \cdots, h_{n}(t)\right)^{T} \in \mathcal{H}$ and $\bar{h}(t)=\left(\bar{h}_{1}(t), \bar{h}_{2}(t), \cdots, \bar{h}_{n}(t)\right)^{T} \in$ $\mathcal{H}$.

\section{$3 \quad$ Main results}

Theorem 3.1 Under conditions (2.4)-(2.6). If

1) there exists a constant $\mu$ such that $\inf _{k=1,2, \cdots}\left\{t_{k}-t_{k-1}\right\} \geq \mu$,

2) there exists constants $p_{i}$ such that $p_{i k} \leq p_{i} \mu$ for $i \in \mathcal{N}$ and $k=1,2, \cdots$,

3) $\vartheta=\sum_{i=1}^{n}\left\{\frac{1}{d_{i}} \max _{j \in \mathcal{N}}\left(\left|a_{i j}\right| l_{j}+\left|b_{i j}\right| k_{j}\right)\right\}+\max _{i \in \mathcal{N}}\left\{p_{i}\left(\frac{1}{d_{i}}+\mu\right)\right\}<1$,

hold, then systems (2.1)-(2.3) are globally exponentially stable.

Proof. The following proof is based on the contraction mapping principle.

Firstly, we will determine a mapping. From (2.1), for $t>1$ and $t \neq t_{k}$, we have

$$
\begin{aligned}
\mathrm{de}^{d_{i} t} x_{i}(t) & =\mathrm{e}^{d_{i} t} \mathrm{~d} x_{i}(t)+d_{i} x_{i}(t) \mathrm{e}^{d_{i} t} \mathrm{~d} t \\
& =\mathrm{e}^{d_{i} t}\left\{\sum_{j=1}^{n} a_{i j} f_{j}\left(x_{j}(t)\right)+\sum_{j=1}^{n} b_{i j} g_{j}\left(x_{j}\left(q_{j} t\right)\right)\right\} \mathrm{d} t,
\end{aligned}
$$

which yield, after integrating from $t_{k-1}+\varepsilon(\varepsilon>0)$ to $t \in\left(t_{k-1}, t_{k}\right)(k=$ $1,2, \cdots)$,

$$
\begin{aligned}
x_{i}(t) \mathrm{e}^{d_{i} t}= & x_{i}\left(t_{k-1}+\varepsilon\right) \mathrm{e}^{d_{i}\left(t_{k-1}+\varepsilon\right)}+\int_{t_{k-1}+\varepsilon}^{t} \mathrm{e}^{d_{i} s}\left\{\sum_{j=1}^{n} a_{i j} f_{j}\left(x_{j}(s)\right)\right. \\
& \left.+\sum_{j=1}^{n} b_{i j} g_{j}\left(x_{j}\left(q_{j} s\right)\right)\right\} \mathrm{d} s .
\end{aligned}
$$


Let $\varepsilon \rightarrow 0$ in (3.1), we have, for $t \in\left(t_{k-1}, t_{k}\right)(k=1,2, \cdots)$,

$$
\begin{aligned}
x_{i}(t) \mathrm{e}^{d_{i} t}= & x_{i}\left(t_{k-1}+\right) \mathrm{e}^{d_{i} t_{k-1}}+\int_{t_{k-1}}^{t} \mathrm{e}^{d_{i} s}\left\{\sum_{j=1}^{n} a_{i j} f_{j}\left(x_{j}(s)\right)\right. \\
& \left.+\sum_{j=1}^{n} b_{i j} g_{j}\left(x_{j}\left(q_{j} s\right)\right)\right\} \mathrm{d} s .
\end{aligned}
$$

Set $t=t_{k}-\varepsilon(\varepsilon>0)$ in (3.2), we get

$$
\begin{gathered}
x_{i}\left(t_{k}-\varepsilon\right) \mathrm{e}^{d_{i}\left(t_{k}-\varepsilon\right)}=x_{i}\left(t_{k-1}+\right) \mathrm{e}^{d_{i} t_{k-1}}+\int_{t_{k-1}}^{t_{k}-\varepsilon} \mathrm{e}^{d_{i} s}\left\{\sum_{j=1}^{n} a_{i j} f_{j}\left(x_{j}(s)\right)\right. \\
\left.+\sum_{j=1}^{n} b_{i j} g_{j}\left(x_{j}\left(q_{j} s\right)\right)\right\} \mathrm{d} s,
\end{gathered}
$$

which generates by letting $\varepsilon \rightarrow 0$

$$
\begin{aligned}
x_{i}\left(t_{k}-\right) \mathrm{e}^{d_{i} t_{k}} & =x_{i}\left(t_{k-1}+\right) \mathrm{e}^{d_{i} t_{k-1}}+\int_{t_{k-1}}^{t_{k}} \mathrm{e}^{d_{i} s}\left\{\sum_{j=1}^{n} a_{i j} f_{j}\left(x_{j}(s)\right)\right. \\
+ & \left.\sum_{j=1}^{n} b_{i j} g_{j}\left(x_{j}\left(q_{j} s\right)\right)\right\} \mathrm{d} s
\end{aligned}
$$

Note $x_{i}\left(t_{k}-0\right)=x_{i}\left(t_{k}\right),(3.3)$ can be rewritten as

$$
\begin{aligned}
x_{i}\left(t_{k}\right) \mathrm{e}^{d_{i} t_{k}} & =x_{i}\left(t_{k-1}+\right) \mathrm{e}^{d_{i} t_{k-1}}+\int_{t_{k-1}}^{t_{k}} \mathrm{e}^{d_{i} s}\left\{\sum_{j=1}^{n} a_{i j} f_{j}\left(x_{j}(s)\right)\right. \\
& \left.+\sum_{j=1}^{n} b_{i j} g_{j}\left(x_{j}\left(q_{j} s\right)\right)\right\} \mathrm{d} s,
\end{aligned}
$$

Combining (3.2) and (3.4), we obtain

$$
x_{i}(t) \mathrm{e}^{d_{i} t}=x_{i}\left(t_{k-1}+\right) \mathrm{e}^{d_{i} t_{k-1}}+\int_{t_{k-1}}^{t} \mathrm{e}^{d_{i} s}\left\{\sum_{j=1}^{n} a_{i j} f_{j}\left(x_{j}(s)\right)+\sum_{j=1}^{n} b_{i j} g_{j}\left(x_{j}\left(q_{j} s\right)\right)\right\} \mathrm{d} s
$$

for $t \in\left(t_{k-1}, t_{k}\right](k=1,2, \cdots)$. Further, 


$$
\begin{aligned}
x_{i}(t) \mathrm{e}^{d_{i} t}= & \left\{x_{i}\left(t_{k-1}\right)+P_{i(k-1)}\left(x_{i}\left(t_{k-1}\right)\right)\right\} \mathrm{e}^{d_{i} t_{k-1}} \\
& +\int_{t_{k-1}}^{t} \mathrm{e}^{d_{i} s}\left\{\sum_{j=1}^{n} a_{i j} f_{j}\left(x_{j}(s)\right)+\sum_{j=1}^{n} b_{i j} g_{j}\left(x_{j}\left(q_{j} s\right)\right)\right\} \mathrm{d} s \\
=x_{i} & \left(t_{k-1}\right) \mathrm{e}^{d_{i} t_{k-1}}+\int_{t_{k-1}}^{t} \mathrm{e}^{d_{i} s}\left\{\sum_{j=1}^{n} a_{i j} f_{j}\left(x_{j}(s)\right)+\sum_{j=1}^{n} b_{i j} g_{j}\left(x_{j}\left(q_{j} s\right)\right)\right\} \mathrm{d} s \\
& +P_{i(k-1)}\left(x_{i}\left(t_{k-1}\right)\right) \mathrm{e}^{d_{i} t_{k-1}}
\end{aligned}
$$

holds for $t \in\left(t_{k-1}, t_{k}\right](k=1,2, \cdots)$. Thus,

$$
\begin{gathered}
x_{i}\left(t_{k-1}\right) \mathrm{e}^{d_{i} t_{k-1}}=x_{i}\left(t_{k-2}\right) \mathrm{e}^{d_{i} t_{k-2}}+\int_{t_{k-2}}^{t_{k-1}} \mathrm{e}^{d_{i} s}\left\{\sum_{j=1}^{n} a_{i j} f_{j}\left(x_{j}(s)\right)\right. \\
\left.+\sum_{j=1}^{n} b_{i j} g_{j}\left(x_{j}\left(q_{j} s\right)\right)\right\} \mathrm{d} s+P_{i(k-2)}\left(x_{i}\left(t_{k-2}\right)\right) \mathrm{e}^{d_{i} t_{k-2}}, \\
\vdots \\
x_{i}\left(t_{2}\right) \mathrm{e}^{d_{i} t_{2}}=x_{i}\left(t_{1}\right) \mathrm{e}^{d_{i} t_{1}}+\int_{t_{1}}^{t_{2}} \mathrm{e}^{d_{i} s}\left\{\sum_{j=1}^{n} a_{i j} f_{j}\left(x_{j}(s)\right)+\sum_{j=1}^{n} b_{i j} g_{j}\left(x_{j}\left(q_{j} s\right)\right)\right\} \mathrm{d} s \\
+P_{i 1}\left(z_{i}\left(t_{1}\right)\right) \mathrm{e}^{d_{i} t_{1}}, \\
x_{i}\left(t_{1}\right) \mathrm{e}^{d_{i} t_{1}}=\varphi_{i}(1) \mathrm{e}^{d_{i}}+\int_{1}^{t_{1}} \mathrm{e}^{d_{i} s}\left\{\sum_{j=1}^{n} a_{i j} f_{j}\left(x_{j}(s)\right)+\sum_{j=1}^{n} b_{i j} g_{j}\left(x_{j}\left(q_{j} s\right)\right)\right\} \mathrm{d} s
\end{gathered}
$$

which produces, for $t>1$,

$$
\begin{gathered}
x_{i}(t)=\varphi_{i}(1) \mathrm{e}^{-d_{i}(t-1)}+\mathrm{e}^{-d_{i} t} \int_{1}^{t} \mathrm{e}^{d_{i} s}\left\{\sum_{j=1}^{n} a_{i j} f_{j}\left(x_{j}(s)\right)+\sum_{j=1}^{n} b_{i j} g_{j}\left(x_{j}\left(q_{j} s\right)\right)\right\} \mathrm{d} s \\
+\mathrm{e}^{-d_{i} t} \sum_{1<t_{k}<t}\left\{P_{i k}\left(x_{i}\left(t_{k}\right)\right) \mathrm{e}^{d_{i} t_{k}}\right\} .
\end{gathered}
$$

Note $x_{i}(1)=\varphi_{i}(1)$ in (3.5), we define the following operator $\pi$ acting on $\mathcal{H}$ for $x(t)=\left(x_{1}(t), x_{2}(t), \cdots, x_{n}(t)\right)^{T} \in \mathcal{H}$ :

$$
\pi(x)(t)=\left(\pi\left(x_{1}\right)(t), \pi\left(x_{2}\right)(t), \cdots, \pi\left(x_{n}\right)(t)\right),
$$

where $\pi\left(x_{i}\right)(t):[q, \infty) \rightarrow \mathbb{R}(i \in \mathcal{N})$, and

$$
\begin{aligned}
\pi\left(x_{i}\right)(t)= & \varphi_{i}(1) \mathrm{e}^{-d_{i}(t-1)}+\mathrm{e}^{-d_{i} t} \int_{1}^{t} \mathrm{e}^{d_{i} s}\left\{\sum_{j=1}^{n} a_{i j} f_{j}\left(x_{j}(s)\right)\right. \\
& \left.+\sum_{j=1}^{n} b_{i j} g_{j}\left(x_{j}\left(q_{j} s\right)\right)\right\} \mathrm{d} s+\mathrm{e}^{-d_{i} t} \sum_{0<t_{k}<t}\left\{P_{i k}\left(x_{i}\left(t_{k}\right)\right) \mathrm{e}^{-d_{i} t_{k}}\right\},
\end{aligned}
$$


for $t \geq 1$ and $\pi\left(x_{i}\right)(s)=\varphi_{i}(s)$ on $s \in[q, 1]$.

Secondly, we will prove that $\pi(\mathcal{H}) \subset \mathcal{H}$. Taking $x_{i}(t) \in \mathcal{H}_{i}(i \in \mathcal{N})$, we will testify $\pi\left(x_{i}\right)(t) \subset \mathcal{H}_{i}$.

Firstly, by $\pi\left(x_{i}\right)(s)=\varphi_{i}(s)$ on $s \in[q, 1]$. Then, for a fixed time $t>1$, it follows from (3.6) that

$$
\pi\left(x_{i}\right)(t+r)-\pi\left(x_{i}\right)(t)=Q_{1}+Q_{2}+Q_{3}+Q_{4},
$$

where

$$
\begin{aligned}
& Q_{1}=\varphi_{i}(1) \mathrm{e}^{-d_{i}(t-1+r)}-\varphi_{i}(1) \mathrm{e}^{-d_{i}(t-1)}, \\
& Q_{2}=\mathrm{e}^{-d_{i}(t+r)} \int_{1}^{t+r} \mathrm{e}^{d_{i} s} \sum_{j=1}^{n} a_{i j} f_{j}\left(x_{j}(s)\right)-\mathrm{e}^{-d_{i} t} \int_{1}^{t} \mathrm{e}^{d_{i} s} \sum_{j=1}^{n} a_{i j} f_{j}\left(x_{j}(s)\right) \mathrm{d} s, \\
& Q_{3}=\mathrm{e}^{-d_{i}(t+r)} \int_{1}^{t+r} \mathrm{e}^{d_{i} s} \sum_{j=1}^{n} b_{i j} g_{j}\left(x_{j}\left(q_{j} s\right)\right) \mathrm{d} s-\mathrm{e}^{-d_{i} t} \int_{1}^{t} \mathrm{e}^{d_{i} s} \sum_{j=1}^{n} b_{i j} g_{j}\left(x_{j}\left(q_{j} s\right)\right) \mathrm{d} s, \\
& Q_{4}=\mathrm{e}^{-d_{i}(t+r)} \sum_{1<t_{k}<(t+r)} P_{i k}\left(x_{i}\left(t_{k}\right)\right) \mathrm{e}^{d_{i} t_{k}}-\mathrm{e}^{-d_{i} t} \sum_{1<t_{k}<t} P_{i k}\left(x_{i}\left(t_{k}\right)\right) \mathrm{e}^{d_{i} t_{k}} .
\end{aligned}
$$

Since $x_{i}(t) \in \mathcal{H}_{i}$, we know that $x_{i}(t)$ is continuous on $t \neq t_{k}(k=1,2, \cdots)$. Moreover, as $t=t_{k}, \lim _{t \rightarrow t_{k}^{-}} x_{i}(t)$ and $\lim _{t \rightarrow t_{k}^{+}} x_{i}(t)$ exist, and $\lim _{t \rightarrow t_{k}^{-}} x_{i}(t)=$ $x_{i}\left(t_{k}\right)$.

Consequently, it is easy to find that $Q_{i} \rightarrow 0$ as $r \rightarrow 0$ for $i=1,2,3,4$ as $t \neq t_{k}(k=1,2, \cdots)$ in $(3.7)$, and so $\pi\left(x_{i}\right)(t)$ is continuous on the fixed time $t \neq t_{k}(k=1,2, \cdots)$. In addition, it is not difficult to know that $Q_{i} \rightarrow 0$ as $r \rightarrow 0$ for $i=1,2,3$ as $t=t_{k}(k=1,2, \cdots)$ in (3.7). In the meantime, let $r<0$ be small enough, we have

$$
\begin{aligned}
Q_{4} & =\mathrm{e}^{-d_{i}\left(t_{k}+r\right)} \sum_{1<t_{m}<\left(t_{k}+r\right)} P_{i m}\left(x_{i}\left(t_{m}\right)\right) \mathrm{e}^{d_{i} t_{m}}-\mathrm{e}^{-d_{i} t_{k}} \sum_{1<t_{m}<t_{k}} P_{i m}\left(x_{i}\left(t_{m}\right)\right) \mathrm{e}^{d_{i} t_{m}} \\
& =\left(\mathrm{e}^{-d_{i}\left(t_{k}+r\right)}-\mathrm{e}^{-d_{i} t_{k}}\right) \sum_{1<t_{m}<t_{k}} P_{i m}\left(x_{i}\left(t_{m}\right)\right) \mathrm{e}^{d_{i} t_{m}},
\end{aligned}
$$

which implies $\lim _{r \rightarrow 0^{-}} Q_{4}=0$. While let $r>0$ be small enough, we derive 


$$
\begin{aligned}
Q_{4} & =\mathrm{e}^{-d_{i}\left(t_{k}+r\right)} \sum_{1<t_{m}<\left(t_{k}+r\right)} P_{i m}\left(x_{i}\left(t_{m}\right)\right) \mathrm{e}^{d_{i} t_{m}}-\mathrm{e}^{-d_{i} t_{k}} \sum_{1<t_{m}<t_{k}} P_{i m}\left(x_{i}\left(t_{m}\right)\right) \mathrm{e}^{d_{i} t_{m}} \\
& =\mathrm{e}^{-d_{i}\left(t_{k}+r\right)}\left\{\sum_{1<t_{m}<t_{k}}\left\{P_{i m}\left(x_{i}\left(t_{m}\right)\right) \mathrm{e}^{d_{i} t_{m}}\right\}+P_{i k}\left(x_{i}\left(t_{k}\right)\right) \mathrm{e}^{d_{i} t_{k}}\right\} \\
& -\mathrm{e}^{-d_{i} t_{k}} \sum_{1<t_{m}<t_{k}}\left\{P_{i m}\left(x_{i}\left(t_{m}\right)\right) \mathrm{e}^{d_{i} t_{m}}\right\} \\
= & \left(\mathrm{e}^{-d_{i}\left(t_{k}+r\right)}-\mathrm{e}^{-d_{i} t_{k}}\right) \sum_{1<t_{m}<t_{k}}\left\{P_{i m}\left(x_{i}\left(t_{m}\right)\right) \mathrm{e}^{d_{i} t_{m}}\right\}+\mathrm{e}^{-d_{i}\left(t_{k}+r\right)} P_{i k}\left(x_{i}\left(t_{k}\right)\right) \mathrm{e}^{d_{i} t_{k}},
\end{aligned}
$$

which yields $\lim _{r \rightarrow 0^{+}} Q_{4}=P_{i k}\left(x_{i}\left(t_{k}\right)\right)$.

On the basis of the above discussion, we know that $\pi\left(x_{i}\right)(t):[q, \infty) \rightarrow \mathbb{R}$ is continuous on $t \neq t_{k}(k=1,2, \cdots)$, and $\lim _{t \rightarrow t_{k}^{-}} \pi\left(x_{i}\right)(t)$ and $\lim _{t \rightarrow t_{k}^{+}} \pi\left(x_{i}\right)(t)$ exist for $t=t_{k}(k=1,2, \cdots)$; moreover, $\lim _{t \rightarrow t_{k^{-}}} \pi\left(x_{i}\right)(t)=\pi\left(x_{i}\right)\left(t_{k}\right) \neq$ $\lim _{t \rightarrow t_{k^{+}}} \pi\left(x_{i}\right)(t)$. Next, we will prove $\mathrm{e}^{\alpha t} \pi\left(x_{i}\right)(t) \rightarrow 0$ as $t \rightarrow \infty$ for $i \in \mathcal{N}$. First, Obviously, $\lim _{t \rightarrow \infty} \mathrm{e}^{-\left(d_{i}-\alpha\right) t}=0$ for $d_{i}-\alpha>0$. In addition, because of $x_{j}(t) \in \mathcal{H}_{j}$ for $j \in \mathcal{N}$, we know $\lim _{t \rightarrow \infty} \mathrm{e}^{2 \alpha t} x_{j}(t)=0$, thus $\lim _{t \rightarrow \infty} \mathrm{e}^{\alpha t} x_{j}(t)=0$. Then, for any $\varepsilon>0$, there exists a $T_{j}>1$ such that $s \geq T_{j}$, which implies $\left|\mathrm{e}^{\alpha s} x_{j}(s)\right|<\varepsilon$. Choose $T^{*}=\max _{j \in \mathcal{N}}\left\{T_{j}\right\}$. From (2.4), we have

$$
\begin{aligned}
\mathrm{e}^{\alpha t} \mathrm{e}^{-d_{i} t} \int_{1}^{t} \mathrm{e}^{d_{i} s} \sum_{j=1}^{n} a_{i j} f_{j}\left(x_{j}(s)\right) \mathrm{d} s \\
\leq \mathrm{e}^{\alpha t} \mathrm{e}^{-d_{i} t} \int_{1}^{t} \mathrm{e}^{d_{i} s} \sum_{j=1}^{n}\left\{\left|a_{i j}\right| l_{j}\left|x_{j}(s)\right|\right\} \mathrm{d} s \\
=\mathrm{e}^{-\left(d_{i}-\alpha\right) t} \int_{1}^{T^{*}} \mathrm{e}^{\left(d_{i}-\alpha\right) s} \sum_{j=1}^{n}\left\{\left|a_{i j}\right| l_{j}\left|\mathrm{e}^{\alpha s} x_{j}(s)\right|\right\} \mathrm{d} s \\
\quad+\mathrm{e}^{-\left(d_{i}-\alpha\right) t} \int_{T^{*}}^{t} \mathrm{e}^{\left(d_{i}-\alpha\right) s} \sum_{j=1}^{n}\left\{\left|a_{i j}\right| l_{j}\left|\mathrm{e}^{\alpha s} x_{j}(s)\right|\right\} \mathrm{d} s \\
\leq \mathrm{e}^{-\left(d_{i}-\alpha\right) t} \sum_{j=1}^{n}\left\{\left|a_{i j}\right| l_{j} \sup _{s \in\left[1, T^{*}\right]}\left|\mathrm{e}^{\alpha s} x_{j}(s)\right|\right\}\left\{\int_{1}^{T^{*}} \mathrm{e}^{\left(d_{i}-\alpha\right) s} \mathrm{~d} s\right\} \\
\quad+\varepsilon \sum_{j=1}^{n}\left\{\left|a_{i j}\right| l_{j}\right\} \mathrm{e}^{-\left(d_{i}-\alpha\right) t} \int_{T^{*}}^{t} \mathrm{e}^{\left(d_{i}-\alpha\right) s} \mathrm{~d} s \\
\leq \mathrm{e}^{-\left(d_{i}-\alpha\right) t} \sum_{j=1}^{n}\left\{\left|a_{i j}\right| l_{j} \sup _{s \in\left[1, T^{*}\right]}\left|\mathrm{e}^{\alpha s} x_{j}(s)\right|\right\}\left\{\int_{1}^{T^{*}} \mathrm{e}^{\left(d_{i}-\alpha\right) s} \mathrm{~d} s\right\}+\frac{\varepsilon}{d_{i}-\alpha} \sum_{j=1}^{n}\left\{\left|a_{i j}\right| l_{j}\right\}
\end{aligned}
$$

which leads to

$$
\mathrm{e}^{\alpha t} \mathrm{e}^{-d_{i} t} \int_{1}^{t} \mathrm{e}^{d_{i} s} \sum_{j=1}^{n} a_{i j} f_{j}\left(x_{j}(s)\right) \mathrm{d} s \rightarrow 0 \text { as } t \rightarrow \infty \text { for } i \in \mathcal{N} \text {. }
$$

Similarly, since $\lim _{t \rightarrow \infty} \mathrm{e}^{2 \alpha t} x_{j}(t)=0$, for any $\varepsilon>0$, there also exists a $T_{j}^{\prime}>1$ such that $s \geq q T_{j}^{\prime}$ implies $\left|\mathrm{e}^{2 \alpha s} x_{j}(s)\right|<\varepsilon$. Take $\hat{T}=\max _{j \in \mathcal{N}}\left\{T_{j}^{\prime}\right\}$, thus 
$\sup _{s \in[q \hat{T}, t]}\left|\mathrm{e}^{2 \alpha s} x_{j}(s)\right|<\varepsilon$. It follows from (2.5) that

$$
\begin{aligned}
& \mathrm{e}^{\alpha t} \mathrm{e}^{-d_{i} t} \int_{1}^{t} \mathrm{e}^{d_{i} s} \sum_{j=1}^{n} b_{i j} f_{j}\left(x_{j}\left(q_{j} s\right)\right) \mathrm{d} s \\
& \leq \mathrm{e}^{\alpha t} \mathrm{e}^{-d_{i} t} \int_{1}^{t} \mathrm{e}^{d_{i} s} \sum_{j=1}^{n}\left\{\left|b_{i j}\right| k_{j}\left|x_{j}\left(q_{j} s\right)\right|\right\} \mathrm{d} s \\
& =\mathrm{e}^{-\left(d_{i}-\alpha\right) t} \int_{1}^{t} \mathrm{e}^{d_{i} s} \mathrm{e}^{-\alpha q_{j} s} \sum_{j=1}^{n}\left\{\left|b_{i j}\right| k_{j} \mathrm{e}^{\alpha q_{j} s}\left|x_{j}\left(q_{j} s\right)\right|\right\} \mathrm{d} s \\
& \leq \mathrm{e}^{-\left(d_{i}-\alpha\right) t} \int_{1}^{t} \mathrm{e}^{d_{i} s} \mathrm{e}^{-\alpha q s} \sum_{j=1}^{n}\left\{\left|b_{i j}\right| k_{j} \mathrm{e}^{\alpha q_{j} s}\left|x_{j}\left(q_{j} s\right)\right|\right\} \mathrm{d} s \\
& =\mathrm{e}^{-\left(d_{i}-\alpha\right) t} \int_{1}^{t} \mathrm{e}^{\left(d_{i}-\alpha\right) s} \mathrm{e}^{\alpha(1-q) s} \sum_{j=1}^{n}\left\{\left|b_{i j}\right| k_{j} \mathrm{e}^{\alpha q_{j} s}\left|x_{j}\left(q_{j} s\right)\right|\right\} \mathrm{d} s \\
& \leq \mathrm{e}^{-\left(d_{i}-\alpha\right) t} \int_{1}^{t} \mathrm{e}^{\left(d_{i}-\alpha\right) s} \mathrm{e}^{\alpha s} \sum_{j=1}^{n}\left\{\left|b_{i j}\right| k_{j} \mathrm{e}^{\alpha q_{j} s}\left|x_{j}\left(q_{j} s\right)\right|\right\} \mathrm{d} s \\
& \leq \mathrm{e}^{-\left(d_{i}-\alpha\right) t} \int_{1}^{t} \mathrm{e}^{d_{i} s} \sum_{j=1}^{n}\left\{\left|b_{i j}\right| k_{j} \mathrm{e}^{\alpha q_{j} s}\left|x_{j}\left(q_{j} s\right)\right|\right\} \mathrm{d} s \\
& =\mathrm{e}^{-\left(d_{i}-\alpha\right) t} \int_{1}^{\hat{T}} \mathrm{e}^{d_{i} s} \sum_{j=1}^{n}\left\{\left|b_{i j}\right| k_{j} \mathrm{e}^{\alpha q_{j} s}\left|x_{j}\left(q_{j} s\right)\right|\right\} \mathrm{d} s \\
& +\mathrm{e}^{-\left(d_{i}-\alpha\right) t} \int_{\hat{T}}^{t} \mathrm{e}^{d_{i} s} \sum_{j=1}^{n}\left\{\left|b_{i j}\right| k_{j} \mathrm{e}^{\alpha q_{j} s}\left|x_{j}\left(q_{j} s\right)\right|\right\} \mathrm{d} s \\
& \leq \sum_{j=1}^{n}\left\{\left|b_{i j}\right| k_{j} \sup _{s \in[q, \hat{T}]}\left|\mathrm{e}^{\alpha s} x_{j}(s)\right|\right\} \mathrm{e}^{-\left(d_{i}-\alpha\right) t} \int_{1}^{\hat{T}} \mathrm{e}^{d_{i} s} \mathrm{~d} s \\
& +\sum_{j=1}^{n}\left\{\left|b_{i j}\right| k_{j}\right\} \sup _{s \in[q \hat{T}, t]}\left|\mathrm{e}^{\alpha s} x_{j}(s)\right| \mathrm{e}^{-\left(d_{i}-\alpha\right) t} \int_{\hat{T}}^{t} \mathrm{e}^{d_{i} s} \mathrm{~d} s \\
& \leq \mathrm{e}^{-\left(d_{i}-\alpha\right) t} \sum_{j=1}^{n}\left\{\left|b_{i j}\right| k_{j} \sup _{s \in[q, \hat{T}]}\left|\mathrm{e}^{\alpha s} x_{j}(s)\right|\right\} \int_{1}^{\hat{T}} \mathrm{e}^{d_{i} s} \mathrm{~d} s+ \\
& +\sum_{j=1}^{n}\left\{\left|b_{i j}\right| k_{j}\right\} \frac{1}{d_{i}} \mathrm{e}^{\alpha t} \sup _{s \in[q \hat{T}, t]}\left|\mathrm{e}^{\alpha s} x_{j}(s)\right| \\
& \leq \mathrm{e}^{-\left(d_{i}-\alpha\right) t} \sum_{j=1}^{n}\left\{\left|b_{i j}\right| k_{j} \sup _{s \in[q, \hat{T}]}\left|\mathrm{e}^{\alpha s} x_{j}(s)\right|\right\} \int_{1}^{\hat{T}} \mathrm{e}^{d_{i} s} \mathrm{~d} s+ \\
& +\frac{1}{d_{i}} \sum_{j=1}^{n}\left\{\left|b_{i j}\right| k_{j}\right\} \sup _{s \in[q \hat{T}, t]}\left|\mathrm{e}^{2 \alpha s} x_{j}(s)\right| \\
& \leq \mathrm{e}^{-\left(d_{i}-\alpha\right) t} \sum_{j=1}^{n}\left\{\left|b_{i j}\right| k_{j} \sup _{s \in[q, \hat{T}]}\left|\mathrm{e}^{\alpha s} x_{j}(s)\right|\right\} \int_{1}^{\hat{T}} \mathrm{e}^{d_{i} s} \mathrm{~d} s+\frac{\varepsilon}{d_{i}} \sum_{j=1}^{n}\left\{\left|b_{i j}\right| k_{j}\right\},
\end{aligned}
$$

which results in 


$$
\mathrm{e}^{\alpha t} \mathrm{e}^{-d_{i} t} \int_{0}^{t} \mathrm{e}^{d_{i} s} \sum_{j=1}^{n} b_{i j} f_{j}\left(x_{j}\left(q_{j} s\right)\right) \mathrm{d} s \rightarrow 0 \text { as } t \rightarrow \infty \text { for } i \in \mathcal{N} \text {. }
$$

Next, from (2.6), we get that $\left|P_{i k}\left(x_{i}\left(t_{k}\right)\right)\right| \leq p_{i k}\left|x_{i}\left(t_{k}\right)\right|$. Therefore,

$$
\mathrm{e}^{\alpha t} \mathrm{e}^{-d_{i} t} \sum_{1<t_{k}<t}\left\{P_{i k}\left(x_{i}\left(t_{k}\right)\right) \mathrm{e}^{d_{i} t_{k}}\right\} \leq \mathrm{e}^{\alpha t} \mathrm{e}^{-d_{i} t} \sum_{1<t_{k}<t}\left\{p_{i k}\left|x_{i}\left(t_{k}\right)\right| \mathrm{e}^{d_{i} t_{k}}\right\} .
$$

As $x_{i}(t) \in \mathcal{H}_{i}$, we have $\lim _{t \rightarrow \infty} \mathrm{e}^{2 \alpha t} x_{i}(t)=0$, thus $\lim _{t \rightarrow \infty} \mathrm{e}^{\alpha t} x_{i}(t)=0$. Then, for any $\varepsilon>0$, there exists a non-impulsive point $T_{i}>1$ such that $s \geq T_{i}$ implies $\left|\mathrm{e}^{\alpha s} x_{i}(s)\right|<\varepsilon$. Thus, it follows conditions 1) and 2) that

$$
\begin{aligned}
\mathrm{e}^{\alpha t} \mathrm{e}^{-d_{i} t} \sum_{0<t_{k}<t}\left\{p_{i k}\left|x_{i}\left(t_{k}\right)\right| \mathrm{e}^{d_{i} t_{k}}\right\} \\
=\mathrm{e}^{\alpha t} \mathrm{e}^{-d_{i} t}\left\{\sum_{1<t_{k}<T_{i}}\left\{p_{i k}\left|x_{i}\left(t_{k}\right)\right| \mathrm{e}^{d_{i} t_{k}}\right\}+\sum_{T_{i}<t_{k}<t}\left\{p_{i k}\left|x_{i}\left(t_{k}\right)\right| \mathrm{e}^{d_{i} t_{k}}\right\}\right\} \\
\leq \mathrm{e}^{\alpha t} \mathrm{e}^{-d_{i} t} \sum_{1<t_{k}<T_{i}}\left\{p_{i k}\left|x_{i}\left(t_{k}\right)\right| \mathrm{e}^{d_{i} t_{k}}\right\}+\mathrm{e}^{\alpha t} \mathrm{e}^{-d_{i} t} p_{i} \varepsilon \sum_{T_{i}<t_{k}<t}\left\{\mu \mathrm{e}^{-\left(d_{i}-\alpha\right) t_{k}}\right\} \\
\leq \mathrm{e}^{-\left(d_{i}-\alpha\right) t} \sum_{1<t_{k}<T_{i}}\left\{p_{i k}\left|x_{i}\left(t_{k}\right)\right| \mathrm{e}^{d_{i} t_{k}}\right\} \\
\quad+\mathrm{e}^{-\left(d_{i}-\alpha\right) t} p_{i} \varepsilon\left\{\sum_{T_{i}<t_{r}<t_{k}}\left\{\mathrm{e}^{\left(d_{i}-\alpha\right) t_{r}}\left(t_{r+1}-t_{r}\right)\right\}+\mu \mathrm{e}^{\left(d_{i}-\alpha\right) t_{k}}\right\} \\
\leq \mathrm{e}^{-\left(d_{i}-\alpha\right) t} \sum_{1<t_{k}<T_{i}}\left\{p_{i k}\left|x_{i}\left(t_{k}\right)\right| \mathrm{e}^{d_{i} t_{k}}\right\} \\
\quad+\mathrm{e}^{-\left(d_{i}-\alpha\right) t} p_{i} \varepsilon \int_{T_{i}}^{t} \mathrm{e}^{\left(d_{i}-\alpha\right) s} \mathrm{~d} s+\mathrm{e}^{-\left(d_{i}-\alpha\right) t} p_{i} \varepsilon \mu \mathrm{e}^{\left(d_{i}-\alpha\right) t} \\
\leq \mathrm{e}^{-\left(d_{i}-\alpha\right) t} \sum_{1<t_{k}<T_{i}}\left\{p_{i k}\left|x_{i}\left(t_{k}\right)\right| \mathrm{e}^{d_{i} t_{k}}\right\}+\frac{p_{i} \varepsilon}{d_{i}-\alpha}+p_{i} \varepsilon \mu,
\end{aligned}
$$

which produces

$$
\mathrm{e}^{\alpha t} \mathrm{e}^{-d_{i} t} \sum_{1<t_{k}<t}\left\{P_{i k}\left(x_{i}\left(t_{k}\right)\right) \mathrm{e}^{d_{i} t_{k}}\right\} \rightarrow 0 \text { as } t \rightarrow \infty \text {. }
$$

By (3.8)-(3.10), we deduce that $\mathrm{e}^{\alpha t} \pi\left(x_{i}\right)(t) \rightarrow 0$ as $t \rightarrow \infty$. Thus, we conclude that $\pi\left(x_{i}\right)(t) \subset \mathcal{H}_{i}(i \in \mathcal{N})$, which means $\pi(\mathcal{H}) \subset \mathcal{H}$.

Finally, we need to prove $\pi$ is contractive. For $x(t)=\left(x_{1}(t), x_{2}(t), \cdots, x_{n}(t)\right)^{T} \in$ $\mathcal{H}$ and $\bar{x}(t)=\left(\bar{x}_{1}(t), \bar{x}_{2}(t), \cdots, \bar{x}_{n}(t)\right)^{T} \in \mathcal{H}$, we estimate $\left|\pi\left(x_{i}\right)(t)-\pi\left(\bar{x}_{i}\right)(t)\right| \leq$ $J_{1}+J_{2}+J_{3}$, where 


$$
\begin{aligned}
& J_{1}=\mathrm{e}^{-d_{i} t} \int_{1}^{t} \mathrm{e}^{d_{i} s} \sum_{j=1}^{n}\left[\left|a_{i j}\right|\left|f_{j}\left(x_{j}(s)\right)-f_{j}\left(\bar{x}_{j}(s)\right)\right|\right] \mathrm{d} s, \\
& J_{2}=\mathrm{e}^{-d_{i} t} \int_{1}^{t} \mathrm{e}^{d_{i} s} \sum_{j=1}^{n}\left[\left|b_{i j}\right|\left|g_{j}\left(x_{j}\left(q_{j} s\right)\right)-g_{j}\left(\bar{x}_{j}\left(q_{j} s\right)\right)\right|\right] \mathrm{d} s, \\
& J_{3}=\mathrm{e}^{-d_{i} t} \sum_{1<t_{k}<t}\left\{\mathrm{e}^{d_{i} t_{k}}\left|P_{i k}\left(x_{i}\left(t_{k}\right)\right)-P_{i k}\left(\bar{x}_{i}\left(t_{k}\right)\right)\right|\right\} .
\end{aligned}
$$

Note

$$
\begin{aligned}
J_{1} & \leq \mathrm{e}^{-d_{i} t} \int_{1}^{t} \mathrm{e}^{d_{i} s} \sum_{j=1}^{n}\left[\left|a_{i j}\right| l_{j}\left|x_{j}(s)-\bar{x}_{j}(s)\right|\right] \mathrm{d} s \\
& \leq \max _{j \in \mathcal{N}}\left|a_{i j}\right| l_{j} \sum_{j=1}^{n}\left\{\sup _{s \in[1, t]}\left|x_{j}(s)-\bar{x}_{j}(s)\right|\right\} \mathrm{e}^{-d_{i} t} \int_{1}^{t} \mathrm{e}^{d_{i} s} \mathrm{~d} s \\
& \leq \frac{1}{d_{i}} \max _{j \in \mathcal{N}}\left|a_{i j}\right| l_{j} \sum_{j=1}^{n}\left\{\sup _{s \in[1, t]}\left|x_{j}(s)-\bar{x}_{j}(s)\right|\right\} ; \\
J_{2} & \leq \mathrm{e}^{-d_{i} t} \int_{1}^{t} \mathrm{e}^{d_{i} s} \sum_{j=1}^{n}\left[\left|b_{i j}\right| k_{j}\left|x_{j}\left(q_{j} s\right)-\bar{x}_{j}\left(q_{j} s\right)\right|\right] \mathrm{d} s \\
& \leq \max _{j \in \mathcal{N}}\left|b_{i j}\right| k_{j} \sum_{j=1}^{n}\left\{\sup _{s \in[q, t]}\left|x_{j}(s)-\bar{x}_{j}(s)\right|\right\} \mathrm{e}^{-d_{i} t} \int_{1}^{t} \mathrm{e}^{d_{i} s} \mathrm{~d} s \\
& \leq \frac{1}{d_{i}} \max _{j \in \mathcal{N}}\left|b_{i j}\right| k_{j} \sum_{j=1}^{n}\left\{\sup _{s \in[q, t]}\left|x_{j}(s)-\bar{x}_{j}(s)\right|\right\} ;
\end{aligned}
$$

and

$$
\begin{aligned}
J_{3} & \leq \mathrm{e}^{-d_{i} t} \sum_{1<t_{k}<t}\left\{\mathrm{e}^{d_{i} t_{k}} p_{i k}\left|x_{i}\left(t_{k}\right)-\bar{x}_{i}\left(t_{k}\right)\right|\right\} \\
& \leq p_{i} \mathrm{e}^{-d_{i} t} \sup _{s \in[1, t]}\left|x_{i}(s)-\bar{x}_{i}(s)\right| \sum_{1<t_{k}<t}\left\{\mathrm{e}^{d_{i} t_{k}} \mu\right\} \\
& \leq p_{i} \mathrm{e}^{-d_{i} t} \sup _{s \in[1, t]}\left|x_{i}(s)-\bar{x}_{i}(s)\right|\left\{\sum_{1<t_{r}<t_{k}}\left\{\mathrm{e}^{d_{i} t_{r}}\left(t_{r+1}-t_{r}\right)\right\}+\mathrm{e}^{d_{i} t_{k}} \mu\right\} \\
& \leq p_{i} \sup _{s \in[1, t]}\left|x_{i}(s)-\bar{x}_{i}(s)\right| \mathrm{e}^{-d_{i} t}\left\{\int_{1}^{t} \mathrm{e}^{d_{i} s} \mathrm{~d} s+\mathrm{e}^{d_{i} t} \mu\right\} \\
& \leq p_{i}\left(\frac{1}{d_{i}}+\mu\right) \sup _{s \in[1, t]}\left|x_{i}(s)-\bar{x}_{i}(s)\right| .
\end{aligned}
$$

It follows from (3.11)-(3.13) that 


$$
\begin{aligned}
\left|\pi\left(x_{i}\right)(t)-\pi\left(\bar{x}_{i}\right)(t)\right| \leq & \frac{1}{d_{i}} \max _{j \in \mathcal{N}}\left|a_{i j}\right| l_{j} \sum_{j=1}^{n}\left\{\sup _{s \in[1, t]}\left|x_{j}(s)-\bar{x}_{j}(s)\right|\right\} \\
& +\frac{1}{d_{i}} \max _{j \in \mathcal{N}}\left|b_{i j}\right| k_{j} \sum_{j=1}^{n}\left\{\sup _{s \in[q, t]}\left|x_{j}(s)-\bar{x}_{j}(s)\right|\right\} \\
& +p_{i}\left(\frac{1}{d_{i}}+\mu\right) \sup _{s \in[1, t]}\left|x_{i}(s)-\bar{x}_{i}(s)\right|,
\end{aligned}
$$

which implies

$$
\begin{aligned}
\sup _{t \in[q, T]}\left|\pi\left(x_{i}\right)(t)-\pi\left(\bar{x}_{i}\right)(t)\right| \leq & \frac{1}{d_{i}} \max _{j \in \mathcal{N}}\left|a_{i j}\right| l_{j} \sum_{j=1}^{n}\left\{\sup _{s \in[q, T]}\left|x_{j}(s)-\bar{x}_{j}(s)\right|\right\} \\
& +\frac{1}{d_{i}} \max _{j \in \mathcal{N}}\left|b_{i j}\right| k_{j} \sum_{j=1}^{n}\left\{\sup _{s \in[q, T]}\left|x_{j}(s)-\bar{x}_{j}(s)\right|\right\} \\
& +p_{i}\left(\frac{1}{d_{i}}+\mu\right) \sup _{s \in[q, T]}\left|x_{i}(s)-\bar{x}_{i}(s)\right| .
\end{aligned}
$$

Thus,

$$
\sum_{i=1}^{n} \sup _{t \in[q, T]}\left|\pi\left(x_{i}\right)(t)-\pi\left(\bar{x}_{i}\right)(t)\right| \leq \vartheta \sum_{j=1}^{n}\left\{\sup _{s \in[q, T]}\left|x_{j}(s)-\bar{x}_{j}(s)\right|\right\} .
$$

Owing to condition 3), we know that $\pi$ is a contraction mapping, and thus there exists a unique fixed point $\bar{x}(\cdot)$ of $\pi$ in $\mathcal{H}$, which means $\bar{x}(\cdot)$ is the solution to systems (2.1)-(2.3) and satisfies $\mathrm{e}^{\alpha t}\|\bar{x}(\cdot)\| \rightarrow 0$ as $t \rightarrow \infty$.

Let $\mu=1$ in Theorem 3.1, we derive the following theorem.

Theorem 3.2 Under conditions (2.4)-(2.6). If

1) $\inf _{k=1,2, \ldots}\left\{t_{k}-t_{k-1}\right\} \geq 1$,

2) there exists constants $p_{i}$ such that $p_{i k} \leq p_{i}$ for $i \in \mathcal{N}$ and $k=1,2, \cdots$,

3) $\vartheta=\sum_{i=1}^{n}\left\{\frac{1}{d_{i}} \max _{j \in \mathcal{N}}\left(\left|a_{i j}\right| l_{j}+\left|b_{i j}\right| k_{j}\right)\right\}+\max _{i \in \mathcal{N}}\left\{p_{i}\left(\frac{1}{d_{i}}+1\right)\right\}<1$,

hold, then systems (2.1)-(2.3) are globally exponentially stable.

Remark 3.3 In (2.1), if $q_{j}=1, j=1,2, \cdots, n$, then systems (2.1)-(2.3) are a class of impulsive recurrent neural networks without delays. Thus, Theorem$s 3.1$ and 3.2 also apply to the impulsive recurrent neural networks without delays.

Remark 3.4 So far as delay is concerned, in (2.1), time delay function (1$\left.q_{j}\right) t \rightarrow+\infty$ as $q_{j} \neq 1, t \rightarrow+\infty$, so model (2.1) is different from delayed neural network models in [16-20,23,24,37]. Those stability results [16-20,23,24,37] cannot be directly applied to systems (2.1)-(2.3). 
Remark 3.5 The results obtained in this paper and the results in [37] are consistent, however, as for time delays, the research object of the two is completely different. A class of neural networks with bounded time-varying delays is studied in [37], while this paper is devoted to study the neural networks with unbounded proportional delays. The results obtained in this paper extend and improve the results in [37]. On the other hand, the exponential convergence rate of both is different. In [37], the exponential convergence rate is $\alpha<\min _{i \in \mathcal{N}}\left\{d_{i}\right\}$, while the exponential convergence rate is $\alpha<\frac{1}{2} \min _{i \in \mathcal{N}}\left\{d_{i}\right\}$ in this paper, that is, the exponential convergence rate in this paper is less than that in [37].

Remark 3.6 In [25-31], by a nonlinear transformation, a class of neural networks with proportional delays was transformed into a class of neural networks with constant delays and variable coefficients, then by using methods to study, such as constructing Lyapunov functional, linear matrix inequality (LMI), so and on. In this paper, we will not transform a class of neural networks with proportional delays into another neural networks, but directly study the neural networks with proportional delays by using the fixed point theory and some analysis techniques to avoid the difficulty of the new Lyapunov functional structure, or troublesome of LMI. And we obtain the conditions which are simple and easy to verify by using algebraic method.

\section{Example}

In this section, an example will be given showing the effectiveness of the conditions given here.

Example 4.1 Consider a two-neuron impulsive neural networks with proportional delays (2.1) with two modes. The network parameters are given as follows:

$$
D=\left[\begin{array}{ll}
6 & 0 \\
0 & 6
\end{array}\right], A=\left[\begin{array}{cc}
1 & -1 \\
-1 & 1
\end{array}\right], B=\left[\begin{array}{ll}
1 & -2 \\
0 & -1
\end{array}\right], q_{1}=q_{2}=0.5
$$

$f_{j}(s)=g_{j}(s)=\frac{1}{4}(|s+1|-|s-1|), j=1,2, P_{i k}\left(x_{i}\left(t_{k}\right)\right)=0.4 x_{i}\left(t_{k}\right)$ for $i=1,2$ and $k=1,2, \cdots, t_{k}=t_{k-1}+0.5 k(k=1,2, \cdots)$. It is easy to see that $\mu=0.5$ and $l_{j}=k_{j}=\frac{1}{2}$ as well as $p_{i k}=0.4$.

Take $p_{i}=0.8$ and compute

$$
\sum_{i=1}^{2}\left\{\frac{1}{d_{i}} \max _{j=1,2}\left(\left|a_{i j}\right| l_{j}+\left|b_{i j}\right| k_{j}\right)\right\}+\max _{i=1,2}\left\{p_{i}\left(\frac{1}{d_{i}}+\mu\right)\right\}=\frac{171}{180}<1 .
$$


Therefore, it follows from Theorem 3.1 that this impulsive recurrent neural networks with proportional delays (2.1) is globally exponentially stable. Global exponential stability of Example 4.1 without impulse and with impulse in initial value $[1.0,-0.5]^{T}$ can be clearly observed in Fig.1 and Fig.2. From Fig.1 and Fig.2, we can observe that the impulse effects on the stability of neural networks, which makes the network need more time to be stable.

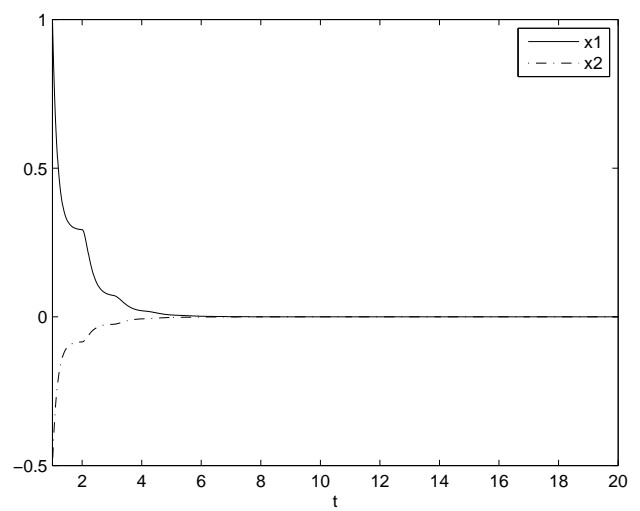

Fig. 1. Global exponential stability of Example 4.1 without impulse in initial value $[1.0,-0.5]^{T}$

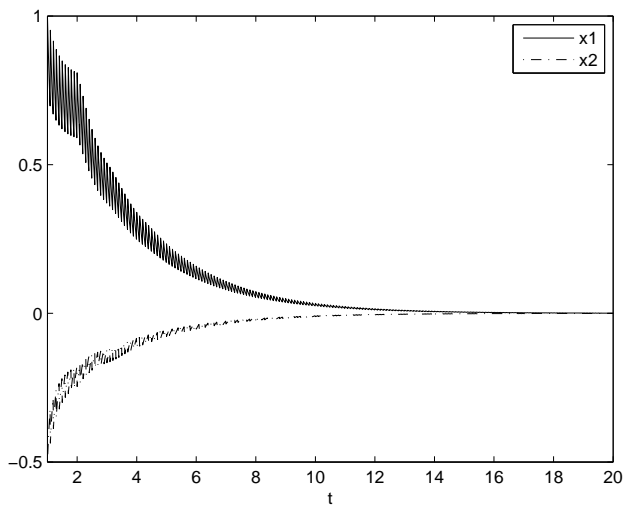

Fig. 2. Global exponential stability of Example 4.1 with impulse in initial value $[1.0,-0.5]^{T}$

\section{Conclusions}

In this paper, without constructing suitable Lyapunov functional, we study the stability of a class of RNNs with proportional delays by using the fixed point theorem, and obtain two new delay-independent sufficient conditions to ensure the existence and uniqueness of a solution and the global exponential 
stability of the systems, which might have an impact on the studying the dissipativity, the existence and stability of periodic solutions, and the synchronization for complex neural networks. The results obtained in this paper extend and improve the research methods in [37]. Different from the prior works, delays here are proportional delays which are unbounded and time-varying. And the nonlinear activation functions are not necessarily differentiable, bounded, monotonic and nondecreasing.

\section{References}

[1] Y. Liu, Z. Wang, X. Liu, Global exponential stability of generalized recurrent neural networks with discrete and distributed delays, Neural Networks 19 (5) (2006) 667-675.

[2] Q. Song, Z. Wang, A delay-dependent LMI approach to dynamics analysis of discrete-time recurrent neural networks with time-varying delays, Physics Letters A 368 (1-2) (2007) 134-145.

[3] Q. Song, Exponential stability of recurrent neural networks with both time-varying delays and general activation functions via LMI approach, Neurocomputing 71 (13-15) (2008) 2823-2830.

[4] K. Ma, L. Yu, W. Zhang, Global exponential stability of cellular neural networks with time-varying discrete and distributed delays, Neurocomputing 72 (10-12) (2009) 2705-2709.

[5] W. Chen, W. Zheng, A new method for complete stability stability analysis of cellular neural networks with time delay, IEEE Transactions on Neural Networks 21 (7) (2010) 1126-1137.

[6] T. Li, A. Song, S. Fei, T. Wang, Delay-derivative-dependent stability for delayed neural networks with unbound distributed delay, IEEE Transactions on Neural Networks 21 (8) (2010) 1365-1371.

[7] Z. Cheng, J. Cao, Hopf bifurcation control for delayed complex networks, Journal of the Franklin Institute, 344 (6) (2007) 846-857.

[8] S. Blythe, X. Mao, X. Liao, Stability of stochastic delay neural networks, Journal of the Franklin Institute 338 (4) (2001) 481 C495.

[9] Q. Zhu, Stochastically asymptotic stability of delayed recurrent neural networks with both Markovian jump parameters and nonlinear disturbances, Journal of the Franklin Institute 347 (8) (2010) 1489-1510.

[10] Y.G. Kao, J.F. Guo, C.H. Wang, X.Q. Sun, Delay-dependent robust exponential stability of Markovian jumping reaction-diffusion Cohen-Grossberg neural networks with mixed delays, Journal of the Franklin Institute 349 (2012) 19721988. 
[11] X.D. Li, C.M. Ding, Q. Zhu, Synchronization of stochastic perturbed chaotic neural networks with mixed delays, Journal of the Franklin Institute 347 (7) (2010) 1266-1280.

[12] H. Shao, Delay dependent stability for recurrent neural networks with timevarying delays, IEEE Transactions on Neural Networks 19 (2008) 1647-1657.

[13] H.G. Zhang, Z.W. Liu, G.B. Huang, Z.S. Wang, Novel weighting delay-based stability criteria for recurrent neural networks with time varying delay, IEEE Transactions on Neural Networks 21 (2010) 91-106.

[14] P. Liu, Delay dependent robust stability analysis for recurrent neural networks with time varying delay, International Journal of Innovative Computing, Information and Control 9 (2013) 3341-3355.

[15] B. Yang, R. Wang, P. Shi, G.M. Dimirovski, New delay-dependent stability criteria for recurrent neural networks with time-varying delays, Neurocomputing 151 (2015) 1414-1422.

[16] J. Qiu, Exponential stability of impulsive neural networks with time-varying delays and reaction-diffusion terms, Neurocomputing 70 (4-6) (2007) 1102-1108.

[17] G.T. Stamov, I.M. Stamova, Almost periodic solution for impulsive neural networks with delay, Applied Mathematical Modelling 31 (7) (2007) 1263-1270.

[18] S. Ahmad, I.M. Stamova, Global exponential stability for impulsive cellular neural networks with time-varying delays, Nonlinear Analysis-Real World Applications 69 (3) (2008) 786-795.

[19] W. Chen, W. Zheng, Global exponential stability of impulsive neural networks with variable delay: an LMI approach, IEEE Transactions on Circuits System-I 56 (6) (2009) 1248-1259.

[20] K. Li, X. Zhang, Z. Li, Global exponential stability of impulsive cellular neural networks with time-varying and distributed delays, Chaos, Solitons and Fractals 41 (3) (2009) 1427-1434.

[21] Q. Zhu, J. Cao, Stability of Markovian jump neural networks with impulse control and time varying delays, Nonlinear Analysis: Real World Applications 13 (5) )2012) 2259-2270.

[22] Q. Zhu, J. Cao, Robust exponential stability of Markovian jump impulsive stochastic Cohen-Grossberg neural networks with mixed time delays, IEEE Transactions on Neural Netwoks 21 (8) (2010) 1314-1325.

[23] Y. Zhang, Q. Luo, Global exponential stability of impulsive delayed reactiondiffusion neural networks via Hardy-Poincarè inequality, Neurocomputing 83 (4) (2012) 198-204.

[24] Y. Zhang, Q. Luo, Novel stability criteria for impulsive delayed reactiondiffusion Cohen-Grossberg neural networks via Hardy-Poincarè inequality, Chaos, Solitons and Fractals 45 (8) (2012) 1033-1040. 
[25] L. Zhou, Dissipativity of a class of cellular neural networks with proportional delays, Nonlinear Dynamics 73 (3) (2013) 1895-1903.

[26] L. Zhou, Delay-dependent exponential stability of cellular neural networks with multi-proportional delays, Neural Processing Letters 38 (3) (2013) 321-346.

[27] L. Zhou, X. Chen, Y. Yang, Asymptotic stability of cellular neural networks with multi-proportional delays, Applied Mathematics and Computation 229 (1) (2014) 457-466.

[28] L. Zhou, Global asymptotic stability of cellular neural networks with proportional delays, Nonlinear Dynamics 77 (1) (2014) 41-47.

[29] L. Zhou, Delay-dependent exponential synchronization of recurrent neural networks with multiple proportional delays, Neural Processing Letters (2014) DOI: $10.1007 / 11063-014-9377-2$.

[30] C. Zheng, N. Li, J. Cao, Matrix measure based stability criteria for highorder networks with proportional delay, Neurcomputing 149 (2015) 11491154.Zheng,Hiena

[31] L.V. Hiena, D.T. Son, Finite-time stability of a class of non-autonomous neural networks with heterogeneous proportional delays, Applied Mathematics and Computation 14 (2015) 14-23.

[32] T.A. Burton, B. Zhang, Fixed points and stability of an integral equation: nonuniqueness, Applied Mathematics Letters 17 (7) (2004) 839-846.

[33] J. Luo, Fixed points and exponential stability of mild solutions of stochastic partial differential equations with delays, Journal of Mathematical Analysis and Applications 342 (2) (2008) 753-760.

[34] J. Luo, T. Taniguchi, Fixed points and stability of stochastic neutral partial differential equations with infinite delays, Stostochastic Analysis and Application 27 (6) (2009) 1163-1173.

[35] J. Luo, Fixed points and exponential stability of stochastic Volterra-Levin equations, Journal of Computational and Applied Mathematics 234 (3) (2010) 934-940.

[36] L, Guo, Q. Zhu, Stability analysis for stochastic Volterra-Levin equations with Poisson jumps: fixed point approach, Journal of Mathematical Physics, 52 (4) (2011) ID 042702, 15 pages.

[37] Y. Zhang, Q. Luo, Global exponential stability of impulsive cellular neural networks with time-varying delays via fixed point theory, Advaces in Difference Equations 23 (2013) doi:10.1186/1687-1847-2013-23.

[38] C. Guo, D. O'Regan, F. Deng, R.P. Agarwal, Fixed points and exponential stability for a stochastic neural cellular neural network, Applied Mathematics Letters 26 (8) (2013) 849-853. 
[39] X. Yang, Q. Zhu, pth moment exponential stability of stochastic partial differential equations with poisson jumps, Asian Journal of control 16 (5) (2014) 1482-1491.

[40] Q. Zhu, J. Cao, Mean-square exponential input-to-state stability of stochastic delayed neural networks, Neurocomputing, 131(5) (2014) 157-163.

[41] D.R. Smart, Fixed point theorems, Cambridge University Press, Cambridge (1980). 